\section{Nonconvulsive status epilepticus associated with leptomeningeal carcinomatosis and positive SOX1 antibodies}

Jeong Yeon Kim, MD; Ga Yeon Kim, MD; Jin Heon Jeong, MD; Sang Ho Kim, MD, PhD

Department of Neurology, Dong-A University Hospital, Busan, Republic of Korea

\section{Journal of Neurocritical \\ Care \\ CASE REPORT}

Received: October 18, 2019

Revised: December 20, 2019

Accepted: December 24, 2019

Corresponding Author:

Sang Ho Kim, MD, PhD

Department of Neurology, Dong-A

University Hospital, 26

Daesingongwon-ro, Seo-gu, Busan

49201, Republic of Korea

Tel: +82-51-240-2962

Fax: +82-51-240-2962

E-mail: shkim1@dau.ac.kr

\begin{abstract}
Background: Nonconvulsive status epilepticus (NCSE) is a disorder with varying prognoses depending on the underlying etiology. It is increasingly recognized as a cause of altered mental status but is difficult to diagnose without continuous electroencephalogram (EEG) monitoring.

Case Report: A 75-year-old woman visited our emergency department with acute altered mental status. A cerebrospinal fluid study showed meningeal carcinomatosis. The EEG showed NCSE. Brain magnetic resonance imaging showed diffuse meningeal enhancement around the brain stem. There were no other masses except suspected primary lung cancer on chest computed tomography. However, no malignant cells were detected in a bronchial washing. Levels of carbohydrate antigen 19-9 (CA19-9), CA125, and CA 15-3 were elevated. Autoimmune antibodies were all negative except for SOX1. The patient was unresponsive to first intrathecal chemotherapy and expired two days later.

Conclusion: This is a case report of NCSE caused by paraneoplastic autoimmune encephalitis associated with SOX1 antibodies.
\end{abstract}

Keywords: Nonconvulsive status epilepticus; Meningeal carcinomatosis; SOX1 antibodies

\section{INTRODUCTION}

Nonconvulsive status epilepticus (NCSE) is a disorder with varying prognoses depending on the underlying etiology. NCSE is increasingly recognized as a cause of altered mental status or coma and accounts for $25 \%$ of all cases of status epilepticus. NCSE is difficult to diagnose without continuous electroencephalogram (EEG) monitoring. NCSE due to paraneoplastic autoimmune encephalitis associated with SOX1 antibodies has been rarely reported.

\section{CASE REPORT}

A 75-year-old woman visited our emergency department with acute altered mental status preceded by a headache, nausea, and vomiting for 3 days. Except for a drowsy mental status, there were no focal neurological signs. Her initial vital signs were wblood pressure $220 / 120 \mathrm{~mm} \mathrm{Hg}$, body temperature $36.1^{\circ} \mathrm{C}$, heart rate 51 per minute, respiratory rate 20 per minute, and $\mathrm{O} 2$ saturation $95 \%$. No abnormalities were noted on brain computed tomography. A cerebrospinal fluid (CSF) analysis revealed elevated open-

(C) 2020 The Korean Neurocritical Care Society

This is an Open Access article distributed under the terms of the Creative Commons Attribution Non-Commercial License (http://creativecommons.org/licenses/by-nc/4.0/) which permits unrestricted noncommercial use, distribution, and reproduction in any medium, provided the original work is properly cited. 
ing pressure $(210 \mathrm{~mm} \mathrm{Hg})$ and mononuclear pleocytosis (60 cells/ $\left.\mathrm{mm}^{3}\right)$ with elevated proteins $(183 \mathrm{mg} / \mathrm{dL})$ and decreased glucose $(30 \mathrm{mg} / \mathrm{dL})$ with a low CSF-to-serum glucose ratio (Table 1). The EEG showed an evolving pattern of rhythmic delta activity ( $>2.5$ $\mathrm{Hz}$ ) with response to intravenous benzodiazepine, a finding compatible with NCSE (Fig. 1). Antiepileptic treatment for NCSE was started with valproic acid 2,000 $\mathrm{mg}$ as a loading dose and $1,000 \mathrm{mg}$ twice daily for maintenance. Burst suppression was achieved on EEG using midazolam $(0.2 \mathrm{mg} / \mathrm{kg} / \mathrm{hr})$ continuous infusion treatment. Brain magnetic resonance imaging showed diffuse meningeal enhancement (Fig. 2). Through further evaluation, a lesion of suspected primary lung cancer was noted on chest computed tomography (Fig. 3), but no masses were noted in the abdomen or pelvis. Bronchoscopy-guided biopsy failed because it was difficult to approach to the lesion's site. Instead, bronchial wash-
Table 1. Cerebrospinal fluid analysis findings

\begin{tabular}{lc}
\hline Variable & Value \\
\hline Intracranial pressure $\left(\mathrm{mmH}_{2} \mathrm{O}\right)$ & 210 \\
Color & Colorless \\
$\mathrm{pH}$ & 7.5 \\
$\mathrm{SG}$ & 1.01 \\
Cell count (WBC) $\left(\mathrm{mm}^{3}\right)$ & 60 \\
Differential count (polymorphonuclear leukocyte) $(\%)$ & - \\
Differential count (lymphocyte) (\%) & 43 \\
Differential count (monocyte) (\%) & 57 \\
Glucose (CSF/serum) & $30 / 158$ \\
Protein (mg/dL) & 183 \\
\hline
\end{tabular}

SG, specific gravity; WBC, white blood cell; CSF, cerebrospinal fluid.
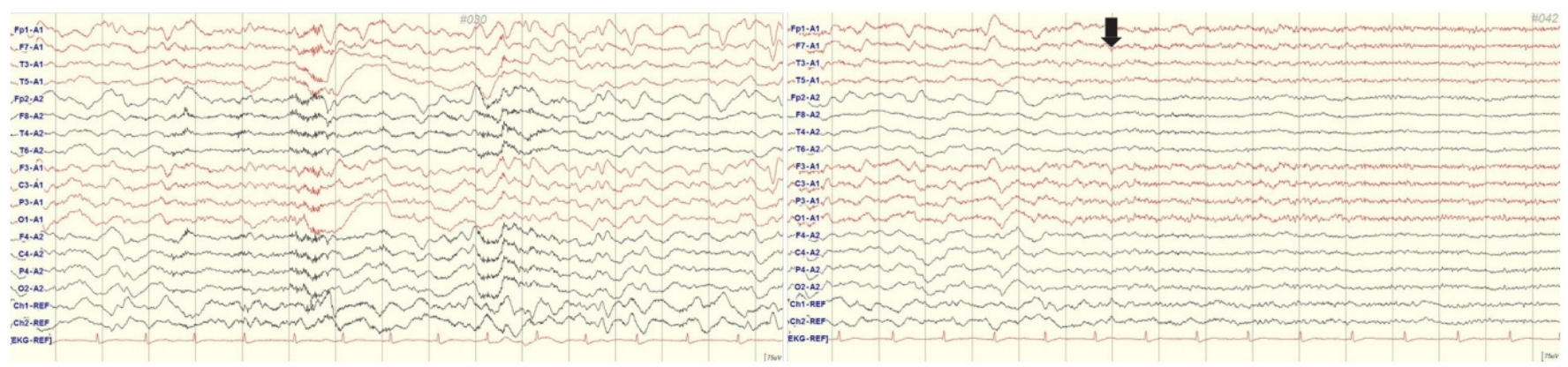

Fig. 1. Initial waking electroencephalography showed semirhythmic delta activity which showed responsiveness (arrow) after 2 mg intravenous lorazepam injection.
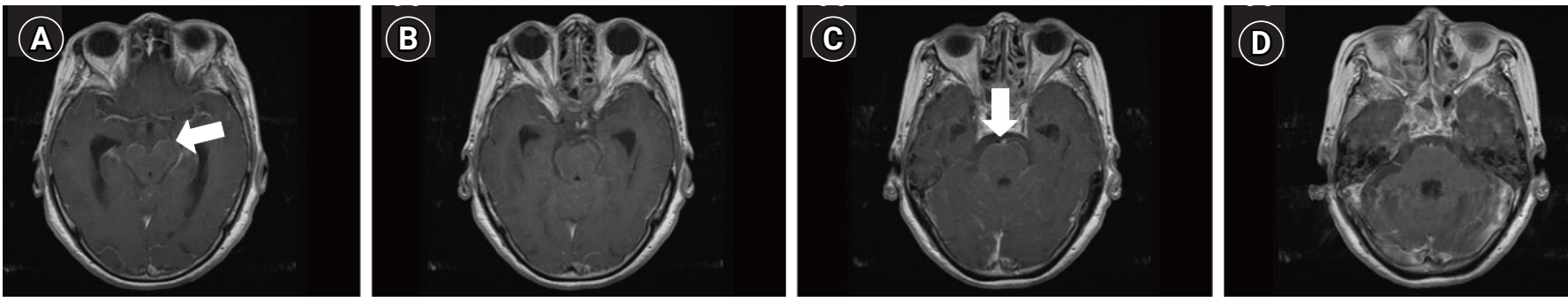

Fig. 2. (A-D) Brain magnetic resonance imaging shows meningeal enhancement around brainstem with focal nodular enhancement (arrows).
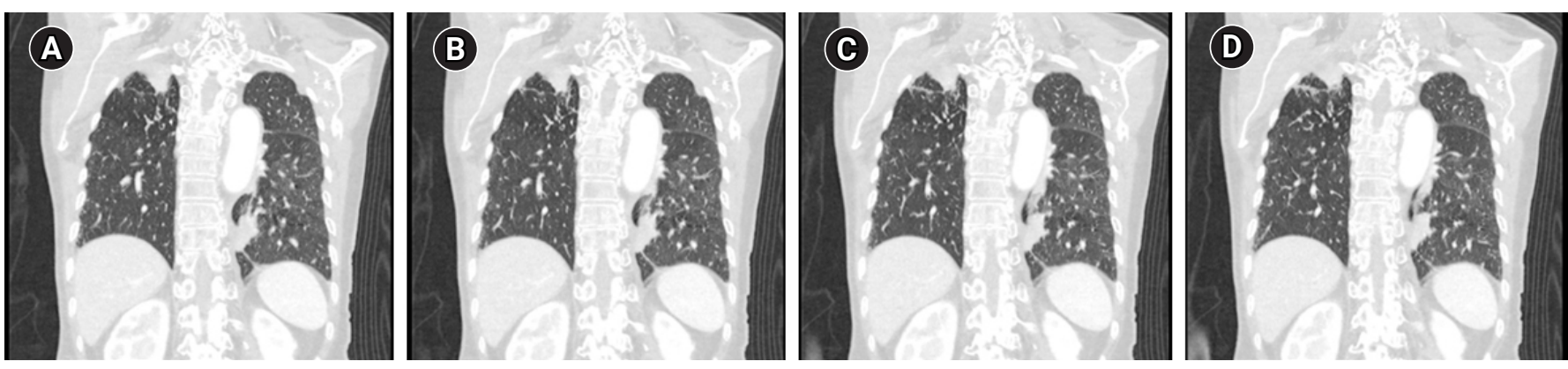

Fig. 3. (A-D) Chest computed tomography showing focal mass like conolidation in left lower lobe posterior basal segment medial aspect. 
ing cytology was done, but no malignant cells were detected. In a tumor marker screening test, elevated levels of carbohydrate antigen 19-9 (CA19-9), CA125, and CA 15-3 were detected (Table 2). CSF cytopathology revealed metastatic adenocarcinoma, which was confirmed as being of lung origin (Fig. 4). Autoimmune synaptic encephalitis antibodies ( $\mathrm{N}$-methyl-D-aspartate receptor [NMDAR], $\alpha$-amino-3-hydroxy-5-methyl-4-isoxazolepropionic acid receptor [AMPA1/2], leucine-rich glioma inactivated 1

Table 2. Paraneoplastic syndrome antibodies, autoimmune synaptic encephalitis antibodies, and serum tumor markers

\begin{tabular}{lc}
\hline Variable & Result \\
\hline Paraneoplastic syndrome antibodies & \\
Hu & $(-)$ \\
Yo & $(-)$ \\
Ri & $(-)$ \\
Ma2 & $(-)$ \\
CV2/CRMP5 & $(-)$ \\
Amphiphysin & $(-)$ \\
Recoverin & $(-)$ \\
S0X1 & $(+)$ \\
Titin & $(-)$ \\
Autoimmune synaptic encephalitis antibodies & \\
NMDAR & \\
AMPA1 & $(-)$ \\
AMPA2 & $(-)$ \\
LGI1 & $(-)$ \\
CASPR2 & $(-)$ \\
GABA-B & $(-)$ \\
AFP (ng/mL) & $(-)$ \\
CEA (ng/mL) & $(-)$ \\
CA19-9 (U/mL) & \\
\hline
\end{tabular}

Hu, ANNA-1, antineuronal nuclear antibody-type 1; Yo, PCA-1, purkinje cell cytoplasmic antibody type $1 ;$ Ri, ANNA-2, , antineuronal nuclear antibody-type 2; Ma2, PNMA2, paraneoplastic antigen MA2; CV2/ CRMP5, collapsing response-mediator protein-5; SOX1, AGNA, antiglial nuclear antibody; NMDAR, N-methyl-D-aspartate receptor; AMPA, a-amino-3-hydroxy-5-methyl-4-isoxazolepropionic acid receptor; LGI1, leucine-rich glioma inactivated 1; CASPR2, contactin-associated proteinlike 2; GABA-B, gamma-aminobutyric acid; AFP, alphafetoprotein; CEA, carcinoembryonic antigen; $\mathrm{CA}$, carbohydrate antigen.
[LGI1], contactin-associated protein-like 2 [CASPR2], gamma-aminobutyric acid [GABA]-B) and paraneoplastic syndrome antibodies (anti-ANNA-1, antineuronal nuclear antibody-type 1 [anti-Hu], anti-PCA-1, Purkinje cell cytoplasmic anti-body type 1 [anti-Yo], ANNA-2, antineuronal nuclear antibody-type2 [anti-Ri], anti-paraneoplastic antigen MA2 [antiPNMA2; Ma2/Ta], collapsing response-mediator protein-5 [CV2/CRMP5], antiamphiphysin, antirecoverin, antititin [MGT-30]) were negative; only SOX1 antibodies were detected (Table 2). Despite the use of midazolam continuous infusion treatment and burst suppression being achieved on EEG, the patient was unresponsive to the first intrathecal chemotherapy (methotrexate $15 \mathrm{mg} /$ day, cytarabine $30 \mathrm{mg} /$ day, cortisol $50 \mathrm{mg}$ / day) and expired suddenly 2 days later due to uncontrolled increased intracranial pressure.

This study was approved by the Institutional Review Board of Dong-A University Hospital (IRB No: DAIHIRB-20-089). Informed consent was waived by the board.

\section{DISCUSSION}

Leptomeningeal metastasis (LM) from cancer was first described in 1870 . The identification of malignant cells on CSF cytology has been the diagnostic gold standard, although its sensitivity is limited. An estimated $10 \%$ to $30 \%$ of patients with solid tumors develop neuraxis metastases, of which $4 \%$ to $15 \%$ represent LM

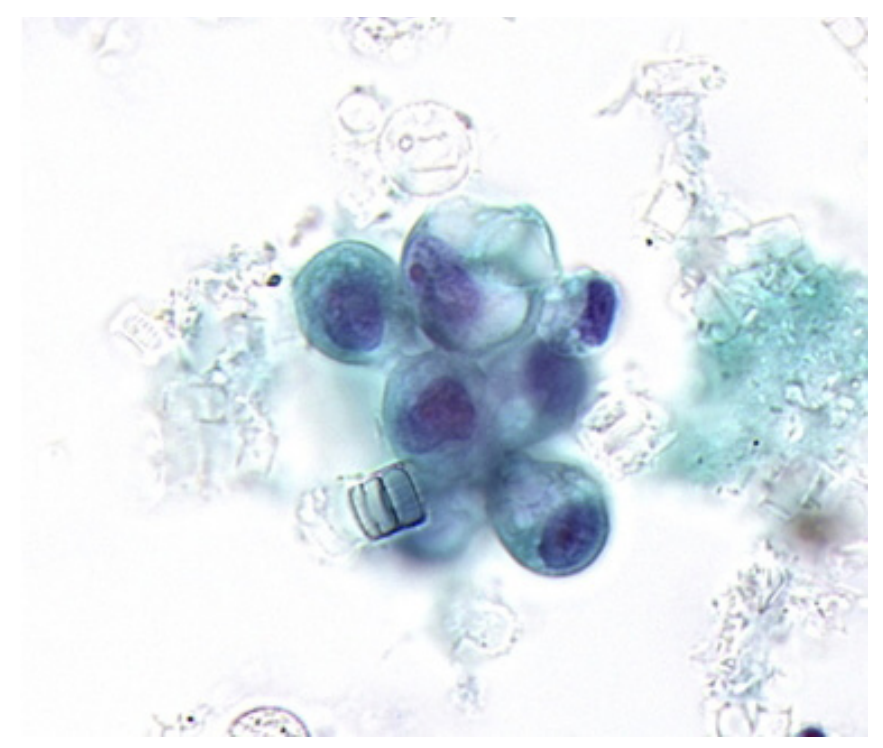

Fig. 4. Cytologic examination of the cerebrospinal fluid shows a few clusters of cells with enlarged eccentric nucleoli, prominent nucleoi and bubbly cytoplasm, suggesting metastatic adenocarcinoma (Papanicolaou stain, $\times 100$ ). 
[1]. Breast tumors, lung tumors, and malignant melanomas are the principal tumors responsible for LM. Adenocarcinoma is the most frequently encountered histological type [2].

The median overall survival of LM patients is 2.4 months ( $95 \%$ confidence interval, 1.9 to 3.1) [3]. The diagnosis and treatment of NCSE usually depend on etiology, EEG findings, and the patient's clinical status. It is not always possible to identify to what extent the electrographic activity contributes to clinical impairment or ongoing neuronal injury.

The underlying causes of NCSE vary and differ according to the patient population being studied. Approximately one-half to two-thirds of affected patients will have a prior history of seizures or epilepsy. NCSE can be the presenting symptom of infectious or autoimmune encephalitis [4-6]. The NCSE in our case could have been caused by paraneoplastic autoimmune encephalitis associated with SOX1 antibodies rather than LM, which may have been difficult to induce NCSE considering its extent and severity.

SOX1 antibodies are rarely detected but have been reported to occur in paraneoplastic neuropathy, Lambert-Eaton syndrome, and unclear forms of neuropathy and ataxia. SOX1 antibodies are often associated with antibodies against voltage-gated calcium channel, Hu, CV2/CRMP5, or amphiphysin. The most common cancer form associated with SOX1 is small cell lung cancer, but other lung cancer forms have also been reported $[7,8]$. In our case, no autoantibodies other than SOX1 were detected.

In conclusion, this case showed NCSE due to paraneoplastic autoimmune encephalitis associated with SOX1 antibodies rather than LM, although primary lung cancer was not confirmed pathologically. Although the NCSE was controlled by a continuous midazolam infusion, the patient did not respond to the first intrathecal chemotherapy and expired 2 days later.

\section{Conflict of interest}

No potential conflict of interest relevant to this article.

\section{ORCID}

Jeong Yeon Kim, https://orcid.org/0000-0003-2789-4852

Ga Yeon Kim, https://orcid.org/0000-0003-4314-7646

Jin Heon Jeong, https://orcid.org/0000-0002-5878-9206

Sang Ho Kim, https://orcid.org/0000-0001-9638-1933

\section{Author contributions}

Conceptualization: SHK. Data curation: JYK and GYK. Visualization \& Writing original draft: JYK. Writing-review editing: GYK, JHJ, and SHK.

\section{REFERENCES}

1. Wasserstrom WR, Glass JP, Posner JB. Diagnosis and treatment of leptomeningeal metastases from solid tumors: experience with 90 patients. Cancer 1982;49:759-72.

2. Chamberlain MC. Neoplastic meningitis. Curr Neurol Neurosci Rep 2008;8:249-58.

3. Clarke JL, Perez HR, Jacks LM, Panageas KS, Deangelis LM. Leptomeningeal metastases in the MRI era. Neurology 2010;74:1449-54.

4. Bayreuther C, Bourg V, Dellamonica J, Borg M, Bernardin G, Thomas P. Complex partial status epilepticus revealing anti-NMDA receptor encephalitis. Epileptic Disord 2009;11:2615.

5. Grativvol RS, Cavalcante WCP, Castro LHM, Nitrini R, Simabukuro MM. Updates in the diagnosis and treatment of paraneoplastic neurologic syndromes. Curr Oncol Rep 2018;20:92.

6. Mantere O, Saarela M, Kieseppä T, Raij T, Mäntylä T, Lindgren $\mathrm{M}$, et al. Anti-neuronal anti-bodies in patients with early psychosis. Schizophr Res 2018;192:404-7.

7. Stich O, Klages E, Bischler P, Jarius S, Rasiah C, Voltz R, et al. SOX1 antibodies in sera from patients with paraneoplastic neurological syndromes. Acta Neurol Scand 2012;125:326-31.

8. Lancaster E, Martinez-Hernandez E, Dalmau J. Encephalitis and antibodies to synaptic and neuronal cell surface proteins. Neurology 2011;77:179-89. 